\title{
La construcción social del cuerpo en las sociedades contemporáneas
}

\section{Ana Martínez Barreiro}

Universidad de A Coruña. Departamento de Sociología y Ciencia Política y de la Administración anamb@udc.es

\section{Resumen}

Este articulo analiza los principales cambios sociales que se han operado sobre la imagen social del cuerpo en la cultura contemporánea. Bajo la perspectiva de la diversidad, se desarrollan diferentes cuestiones que cada vez cobran mayor interés para las ciencias sociales sobre los usos sociales del cuerpo como un objeto de consumo y signo a la vez y el lenguaje del cuerpo. Asimismo, se plantea la controversia social sobre el futuro de los cuerpos, al explorar el papel de la ciencia y la tecnología en las nuevas concepciones acerca del cuerpo, de la vida y la muerte. El interés de este trabajo está en reconocer el carácter profundo de las interconexiones que existen entre la vida social y el cuerpo y la "socialización de la naturaleza».

Palabras clave: cuerpo, ciencia, tecnología, naturaleza, cambio social.

Abstract. The social construction of the body in modern societies

This investigation project studies the principal social changes that have affected the image of the body in contemporary culture. Using the perspective of diversity, a number of issues of growing interest in the field of Social Sciences are discussed about the social uses of the body as both consumer object and symbol, body language and not of the body itself. It also explores the issue of the social controversy surrounding the future of the body, by exploring the role of science and technology in new concepts about the body, life and death. The interest of this project lies in its recognition of the profound nature of the relationships that exist between social life and the body, and the «socialisation of nature».

Key words: body, science, technology, nature, social change.

\section{Sumario}

Introducción

1. Naturaleza y cultura

2. El auge de la cultura somática

3. La política de los cuerpos: poder/sexualidad

4. El cuerpo frente a la corporalidad
5. El cuerpo «habla»

6. El cuerpo como mercancía y signo

7. Cuerpos, ciencia y tecnología

8. A modo de conclusión

Bibliografía 


\section{Introducción}

Este articulo analiza los principales cambios sociales que se han operado sobre la imagen social del cuerpo en la cultura contemporánea. Bajo la perspectiva de la diversidad, se desarrollan diferentes cuestiones que cada vez cobran mayor interés para las ciencias sociales sobre los usos sociales del cuerpo como un objeto de consumo y signo a la vez y el lenguaje del cuerpo. Asimismo, se plantea la controversia social sobre el futuro de los cuerpos, al explorar el papel de la ciencia y la tecnología en las nuevas concepciones acerca del cuerpo, de la vida y la muerte. El interés de este trabajo está en reconocer el carácter profundo de las interconexiones que existen entre la vida social y el cuerpo y la «socialización de la naturaleza» ${ }^{1}$.

\section{Naturaleza y cultura}

En primer lugar, hay que aclarar el distanciamiento intelectual entre naturaleza y cultura en la sociología. Mientras la sociobiología considera el cuerpo con «una base biológica y presocial sobre la cual se fundan las superestructuras del yo y de la sociedad» (Ch. Shilling, 1993: 41), los constructivistas como Douglas, Foucault, Goffman y Turner toman el cuerpo como algo que pertenece a la cultura y no a una identidad biológica. Desde esta perspectiva, el cuerpo es interpretado culturalmente en todas partes, por lo tanto, la biología no se encuentra excluida de la cultura, sino que está dentro de ella. La suposición de que la biología no pertenece a la cultura fue, durante mucho tiempo, una de las razones por las que los teóricos sociales descuidaron el cuerpo como objeto de estudio.

Es importante conocer por qué la teoría social clásica descuidó y reprimió el cuerpo. Turner (1994) ofrece dos razones para justificar el descuido académico del cuerpo. En primer lugar, la teoría social heredó el dualismo cartesiano que daba prioridad a la mente y a sus propiedades de conciencia y de razón sobre sus propiedades de emoción y de pasión. A su vez, la sociología clásica tendió a evitar las explicaciones del mundo social que tenía en cuenta al cuerpo humano, centrándose en el actor humano como un creador de signos y significados. La preocupación sociológica por la historicidad y el orden social en las sociedades modernas no parecía involucrar al cuerpo salvo en las cuestiones ontológicas. La sociología se ha preocupado del yo-sociedad o de su estructura en lugar de la naturaleza-cultura. La otra razón para el olvido del cuerpo es que lo trató como un fenómeno natural, no social y, por consiguiente, no como un objeto legítimo para la investigación sociológica.

1. Expresión que hace referencia al hecho de que ciertos fenómenos que antes eran naturales, o que venían dados por la naturaleza, ahora tienen un carácter social, es decir, que dependen de nuestras propias decisiones. La reproducción humana es un ejemplo de ello. Las transfusiones sanguíneas, los trasplantes de órganos, la procreaciones artificiales, las fecundaciones in vitro, las experimentaciones en seres humanos, las manipulaciones genéticas son otros ejemplos. Cfr. Anthony Giddens (1998: 167). 
La historia y la antropología han influido especialmente en la legitimidad del cuerpo como objeto de estudio social. El cuerpo tiene una historia y ésta ha ayudado a respaldar el cuerpo como objeto primordial de la teoría social; en este sentido han trabajado Elias (1988), Feher y otros (1991), Laquer y Gallagher (1987), Laquer y Bourgois (1992) y Sennett ${ }^{2}$ (1997), representantes de la escuela anglosajona. Norbert Elias (1988) señala las formas en que nuestra comprensión y nuestras experiencias modernas sobre el cuerpo son históricamente específicas y surgen de procesos sociales y psicológicos que se remontan al siglo XVI. Examina de qué modo los desarrollos históricos, como la centralización cada vez mayor del poder en manos de un número más reducido de señores con la aparición de la aristocracia y cortes reales, sirvieron para frenar la violencia entre las personas y los grupos e inducir a un mayor control social sobre las emociones y una mayor conciencia de uno mismo como «individuo» en un cuerpo. Los nuevos códigos de conducta promovieron la idea de que el propio éxito o fracaso dependían de las buenas maneras. En este aspecto, el cuerpo se muestra como portador de la posición social, tema posteriormente investigado en la cultura contemporánea por Bourdieu. Dentro de la Escuela Historiográfica Francesa es interesante desatacar el estudio de Philippe Ariés (1987) sobre la infancia, que se refiere a las vestimentas y también al tamaño del cuerpo representado en los lienzos. Flandrin (1984) desarrolla temas sobre el amor y sobre el sexo. Sin olvidar la historia del control de los nacimientos, de Alfred Sauvy (1972).

La antropología también ha influido de forma decisiva a la hora de legitimar el cuerpo como objeto de estudio social. Turner (1994) ha dado cuatro razones sobre ello. En primer lugar, la antropología se ocupó de cuestiones ontológicas y también ha estado interesada por la dicotomía entre naturaleza y cultura; esto le llevó a considerar el modo en que el cuerpo, como objeto de la naturaleza, fue mediado por la cultura. La segunda característica de la antropología fue su preocupación por las necesidades y por cómo éstas son afrontadas por la cultura, además de señalar que hay dos clases más de áreas de interés que se centran en el cuerpo como entidad simbólica. Concretamente, en las obras de Mary Douglas (1988, 1979b, 1991), el cuerpo se considera como un sistema de clasificación primario para las culturas, medio a través del cual se representan y se manejan los conceptos de orden y desorden. Por su parte, Marcel Mauss arguye que la cultura da forma al cuerpo y describe con detalle lo que él denomina las «técnicas del cuerpo»: «El modo en que de sociedad en sociedad los seres humanos saben cómo usar sus cuerpos» (Mauss, 1973: 70). Estas «técnicas corporales» son un medio importante para la socialización de los individuos en la cultura; a través de ellas y de su cuerpo, un individuo llega a conocer una cultura y a vivir en ella.

Mary Douglas también ha reconocido el cuerpo como un objeto natural moldeado por las fuerzas sociales. Para esta antropóloga, existen dos cuerpos:

2. Sennett (1997) ha explorado la cambiante relación del cuerpo humano con su entorno construido. 
el «cuerpo físico» y el «cuerpo social». Y afirma que «el cuerpo social restringe el modo en que se percibe el cuerpo físico» (Douglas, 1988: 93). Las propiedades fisiológicas del cuerpo son, pues, el punto de partida para la cultura que hace de mediadora y las traduce en símbolos significativos: «en un sistema de símbolos naturales» (1988: 12). Esto significa que el cuerpo es un medio de expresión altamente restringido, puesto que está muy mediatizado por la cultura y expresa la presión social que tiene que soportar. La situación social se impone en el cuerpo y lo ciñe a actuar de formas concretas, así, el cuerpo se convierte en un símbolo de la situación. Douglas (1979b) utiliza el ejemplo de la risa para ilustrarlo. La risa es una función fisiológica que empieza en la cara, pero puede afectar a todo el cuerpo. No obstante, la situación social determina el grado en que el cuerpo se puede reír. Cuantas menos restricciones, más libre está el cuerpo para reír en voz alta. De este modo, el cuerpo se expresa simbólicamente y se convierte en un símbolo de la situación. Según esta idea, el cuerpo trasmite información sobre la situación; cuanto más tradicional sea el lugar, más formal será y mayores serán las presiones que se ejerzan sobre el mismo ${ }^{3}$.

Tanto la historia como la antropología desarrollan una teoría social del cuerpo en las sociedades premodernas. Tal y como expone la cuestión Turner, el cuerpo ofrece de por sí una amplia superficie apropiada para exhibir públicamente marcas de posición familiar, rango social, afiliación tribal y religiosa, edad, sexo. Esta contingencia la aprovechan al máximo las sociedades premodernas fijando de modo permanente los atributos sociales de los neófitos por medio de ceremonias de rituales que implican a menudo alguna transformación física del cuerpo. En las sociedades contemporáneas, aunque los ritos de iniciación no desaparecen, pierden fuerza en su específico cometido de delimitar grupos sociales, tornándose tan mutables como las propias aspiraciones del hombre de ahora. Así, el tatuaje o el piercing son hoy en día uno de los caprichos más relativos a la moda que un aspecto necesario de la cultura religiosa o de la estratificación social (Turner, 1994: 15). Sin embargo, entre los jóvenes aún se utiliza como símbolo de pertenencia a una «tribu» urbana (Brain, 1979; Polhemus, 1978).

Mientras la antropología y la historia han influido en sugerir cómo el cuerpo ha sido moldeado por la cultura, Michel Foucault, a diferencia de los teóricos sociales clásicos que ignoran o reprimen el cuerpo, demuestra la importancia del cuerpo en la teoría social, lo que contribuye a la inauguración de la sociología del cuerpo. No obstante, recientemente, la teoría social ha reevaluado la importancia del cuerpo, no solamente en la teoría social feminista, sino en términos de análisis de clase y consumo a través de los trabajos de Baudrillard y Bourdieu. La perspectiva interaccionista y fenomenológica de Goffman y Merleau-Ponty han producido una conciencia sociológica de la

3. Este enfoque del cuerpo como símbolo ha conducido a Turner (1989) y a Shilling (1993) a considerar el trabajo de Douglas no tanto una antropología del cuerpo, sino «una antropología del simbolismo del riesgo y de ubicación y estratificación social» (Shilling, 1993: 7). 
significación simbólica del cuerpo en el orden interactivo, sin olvidar el discurso del lenguaje corporal en el análisis del comportamiento microsocial. Y, más recientemente, hay que destacar el papel de la ciencia y la tecnología sobre el futuro de los cuerpos. Ahora bien, para entender el desarrollo de la teoría social del cuerpo en el siglo XX y comienzo del XXI, antes hay que explicar los cambios sociales que han contribuido a situar el cuerpo en un lugar central.

\section{El auge de la cultura somática}

Actualmente, el cuerpo se ha convertido en blanco de múltiples atenciones y es, al mismo tiempo, objetivo de grandes inversiones. La presentación y representación del «yo», la fachada goffmaniana, ha ganado una especial relevancia en relación con los nuevos estilos de vida y el retorno del mito de la eterna juventud. Las prácticas y los saberes son promovidos por múltiples especialistas, como los estilistas, los médicos, los publicistas y los esteticistas, que han contribuido a crear o definir y legitimar los nuevos códigos éticos y estéticos de los usos sociales del cuerpo. Todo este nuevo interés que despierta el cuerpo está estrechamente ligado a transformaciones sociales profundas, a cambios en el modo de producción y en las formas de relación, como también a la emergencia de nuevas formas de dominación. A este respecto, voy a enumerar una serie de razones que explican el surgimiento del cuerpo en las sociedades modernas: en primer lugar, el pensamiento feminista ha cuestionado el tema del cuerpo al criticar el determinismo del cuerpo sexuado y replantear el problema de la discriminación en términos de género. En segundo lugar, con la exaltación de la cultura consumista el cuerpo se transforma en mercancía y pasa a ser el medio principal de producción y distribución de la sociedad de consumo. Así, su mantenimiento, reproducción y representación se convierten en temas centrales en la sociedad de consumo (Baudrillard, 1974; Featherston, 1991a). En tercer lugar, el fuerte cambio demográfico que supone el envejecimiento de la población junto con las modificaciones de la medicina moderna elevan a una significación peculiar la cuestión de la corporificación. La aparición de las medicinas alternativas como la naturista, la homeopática, la aromaterapia, entre otras, llevan a una nueva relación con el cuerpo y a una redefinición del concepto de enfermedad y envejecimiento. El envejecimiento de las poblaciones se ha convertido en un hecho de interés político y económico internacional, porque las implicaciones económicas para el mercado de trabajo, los costes de jubilación, previsiones médicas y alojamiento de mayores se consideran algo negativo (Markides y Cooper, 1987). Es útil ver el hecho del envejecimiento en el contexto límite de los modernos cambios médicos. El envejecimiento de la población es, en parte pero no en su totalidad, consecuencia de los adelantos médicos, que han sido consecuencia, a su vez, de las mejoras en los estándares de vida. El aumento de las expectativas de vida es la dimensión de un escenario más amplio que incluye la inseminación artificial, los trasplantes coronarios, la microcirugía y los avances farmacológicos. En cuarto lugar, con la progresiva secularización de la sociedad, el rechazo y la restric- 
ción del deseo ya no constituyen un tema central en la cultura predominante. La secularización actual de la sociedad facilita la mercantilización y el comercialismo del cuerpo. Muchos de estos avances los anticipó Daniel Bell (1994), quien, en Las contradicciones culturales del capitalismo, sostuvo que había una importante tensión entre los valores ascéticos, en declive, del lugar de trabajo y la creciente importancia de la sensibilidad y el hedonismo originados por el ocio. Este mismo autor, en su obra El advenimiento de la sociedad post-industrial (1991), observa el auge de nuevos valores, como la libertad sin restricciones, el reforzamiento del yo individual y el rechazo de la disciplina. Se trata de lo que R. Inglehar (1991) denomina la "emergencia de valores postmateriales», entre los que se encuentra la autoexpresión y la calidad de vida. Finalmente, la crisis de la modernidad también ha vuelto más central al cuerpo. En este sentido, la ecología y la defensa del medio ambiente han contribuido a un nuevo discurso del cuerpo en relación con la sociedad y la naturaleza: la «socialización de la naturaleza» (Giddens, 1995: 282). En la actualidad, una de las cuestiones fundamentales es: ¿cómo sobreviviremos y bajo qué condiciones? En suma, nuestra corporificación es también fundamental desde un punto de vista político.

\section{La política de los cuerpos: poder/sexualidad}

Foucault estaba especialmente interesado en estudiar los efectos del poder sobre el cuerpo y coloca al cuerpo humano en el centro del escenario, al considerar el modo en que las disciplinas emergentes de la modernidad estaban principalmente enfocadas en la actuación de los cuerpos individuales y de las poblaciones. Este interés le llevó a la construcción de una micro política de regulación del cuerpo y una macro política de vigilancia de las poblaciones. En varias de sus obras investiga el origen y desarrollo de las instituciones modernas, y cómo a través de ellas se ejerce el control de los cuerpos y, por tanto, de las personas ${ }^{4}$. En su obra Vigilar y castigar (2000), avanza la idea sobre una "política del cuerpo». Cuando habla del "cuerpo de los condenados» afirma que el cuerpo está directamente inmerso en el campo político, donde las relaciones de poder que operan sobre él, le obligan a efectuar unas ceremonias, y le exigen unos signos. Y, cuando habla de los "cuerpos dóciles», señala que es dócil un cuerpo «que puede ser sometido, que puede ser utilizado, que puede ser trasformado y perfeccionado», poniendo el ejemplo del soldado de comienzos del siglo XVII, este autor desarrolla la "disciplina» como el arte de hacer obediente al cuerpo humano en las instituciones militares, médicas, escolares e industriales, y es donde se construye lo que el autor denomina la «microfísica del poder», y analiza la distribución de los individuos en el espacio, por zonas y rangos, así como el control del empleo del tiempo y el gesto eficaz. De esta

4. En la Historia de la sexualidad (1995) de Foucault queda patente el «componente social» de las conductas corporales y sexuales. 
forma, muestra cómo las disciplinas están destinadas a fabricar sujetos dóciles y útiles, mientras que desde el siglo XVIII hasta principios del siglo XIX se creía que la inversión en el cuerpo por parte del poder tenía que ser fuerte, laboriosa, meticulosa y constante, de ahí los regímenes disciplinarios formidables que uno encuentra en las escuelas, los hospitales y los cuarteles. El objetivo de estas disciplinas es incrementar la utilidad del cuerpo y aumentar su fuerza. Sin embargo, desde mediados del siglo XIX hasta el XX, Foucault se da cuenta de que este poder tan pesado no es tan indispensable como parecía, que las sociedades industriales pueden contentarse con un poder sobre el cuerpo mucho más relajado. Se descubre entonces que los controles sobre los cuerpos pueden atenuarse y adoptar otras formas. Nos encontramos, así, ante un nuevo tipo de dominación que no se adecua a las formas de legitimidad acuñadas por Max Weber (tradicional, carismática y legal-racional), sino que se fundamenta sobre todo en una legitimidad de carácter técnico-científico o legitimidad en relación con el poder médico y la medicalización.

Desde la perspectiva del materialismo histórico, Jean-Mari Brohm (1975, 1982), sociólogo crítico del deporte, ha desarrollado su reflexión sobre la "condición política del cuerpo». Según este autor, el humanismo del cuerpo que se vive en la actualidad no es más que una expresión de las exigencias del sistema capitalista, pues el hombre seguirá siendo un apéndice de la máquina (términos de Marx), ya que la lógica del cuerpo sigue siendo la lógica del rendimiento. Brohm habla de un doble proceso de «sublimación represiva» o proceso de sometimiento del individuo mediante la represión de los instintos, donde las satisfacciones acordadas (el erotismo, el exhibicionismo del cuerpo, el ejercicio corporal) son satisfacciones "sustitutas» totalmente integradas en el orden establecido. Se trata, según él, de elementos pseudoliberadores (un nuevo opio) que no modifican la condición fundamental que "procurará» la libertad a los individuos, la única filosofía vital posible para quienes carecen de futuro. Esta es una visión omnipotente del aparato capitalista, en la que el cuerpo es fuente de explotación, manipulado por falsas técnicas de la felicidad, entre las cuales se encuentran el culto al cuerpo y al ejercicio físico.

La explicación de Foucault sobre el cuerpo y su relación con el poder es problemática para feministas como McNay (1992) y Ramazanoglu (1993), porque no tienen presente la cuestión del género, que es vital para cualquier explicación del cuerpo y sobre cómo éste se ve manipulado por el poder. Tal como arguye McNay (1992), no sólo el género es la diferencia más fundamental entre los cuerpos, sino que el poder no es equitativo respecto a los cuerpos femeninos y los masculinos: la dominación patriarcal del cuerpo de la mujer. Dentro de la concepción política del cuerpo, pero desde la teoría feminista, diversas autoras han realizado una crítica a la utilización del cuerpo de las mujeres como objetos, signos y mercancías en la sociedad actual. Simone de Beauvoir, en su libro El segundo sexo, denuncia la manipulación del aspecto físico de la mujer y su utilización como «objeto erótico ideal». Entre nosotros, Ángeles Duran (1988) ha estudiado la discrepancia genérica en el conocimiento 
y uso del propio cuerpo. El cuerpo es, por excelencia, lugar de cultura, de socialización, con normas distintas para cada uno de los géneros. Tal como señala la autora, "las normas que se refieren al campo de las mujeres son más estrictas y móviles que las referidas al cuerpo de los hombres, precisamente por su definición cultural de cuerpo/objeto o cuerpo deseado». El cuerpo de las mujeres debe ser bello y al mismo tiempo fértil; es, sobre todo, un cuerpo para los demás. Aunque actualmente este modelo ya no es el único existente; ahora se dan, junto a este modelo tradicional de mujer, sobre todo en las generaciones más jóvenes, otras normas de comportamiento caracterizadas por el individualismo y la búsqueda del bienestar personal. Asimismo, el movimiento feminista ha desarrollo una reflexión sobre el cuerpo de la mujer objeto de represión, de escándalo, de explotación, y sobre los mitos de impureza en la mujer desde el cristianismo y el judaísmo. En este sentido, desde algunas corrientes feministas se reivindica la elaboración, por parte de las mujeres, de un nuevo lenguaje corporal (Irigaray, 1977, 1992). También son numerosos los análisis específicos sobre el sometimiento del cuerpo de las mujeres a la institución médica, como el del colectivo de Mujeres de Boston en su libro clásico Nuestros cuerpos, nuestras vidas (1984), a los requisitos de belleza a los que antes aludíamos, entre otros.

No es posible desarrollar aquí todas estas aportaciones porque desbordaría los limites de este trabajo, no obstante, explicaré brevemente las prácticas disciplinarias que engendran los cuerpos dóciles de las mujeres. Las mujeres están sujetas a muchas prácticas disciplinarias que producen un tipo de cuerpo típicamente femenino. $Y$ es que, al parecer, la feminidad es un artificio, es una construcción social: «es una forma de aplicar y reaplicar las normas de género que revisten otros tantos estilos de cuerpos». Entre las muchas técnicas corporales que producen un cuerpo más femenino se pueden distinguir tres: a) aquéllas que pretenden conseguir un cuerpo de cierto tamaño y configuración, como son: la cirugía estética, los regímenes, las dietas, cuyo extremo máximo se encuentra en la bulimia y la anorexia nerviosa (enfermedad femenina entre los 14 y los 26 años); b) aquellas técnicas que tienen como objetivo conseguir una forma de expresión corporal femenina a través de la forma de moverse, en la mirada y en los gestos, y, por último, c) aquéllas que están dirigidas a mostrar un cuerpo como una superficie decorativa: depilación, maquillaje y adornos (Lee Barty, 1994: 63-92). Todas estas prácticas disciplinarias o técnicas corporales femeninas funcionan como un sistema de micropoderes, pues son esencialmente desigualitarias y asimétricas. Sin embargo, lo más importante de todo este proceso es que este poder no sólo está en los propios agentes de socialización (familia, escuela, medios de comunicación), sino en todas partes y en ninguna a la vez, de tal forma que la ausencia de una estructura formal o institución crea la impresión de que la producción de la feminidad es voluntaria y natural. En suma, lo que pretenden estas disciplinas corporales femeninas es crear compañeras dóciles y obedientes o cuerpos dóciles y obedientes, igual que los cuerpos dóciles y obedientes de los que hablaba Foucault. 


\section{El cuerpo frente a la corporalidad}

La mayoría de los estudios definen nuestro cuerpo como algo objetivo, concreto, que se puede medir con límites precisos; sin embargo, lo que llamamos «esquema corporal» es la idea que tenemos del mismo, por lo tanto es algo subjetivo y sujeto a posibles modificaciones. La corporalidad se constituye en un instrumento de expresión de nuestra propia personalidad, también nos sirve para tomar contacto con el exterior, comparándonos con otros cuerpos y objetos, por lo que se puede hablar, dentro del esquema corporal, del cuerpo objeto, es decir, de la representación aislada que nos hacemos de nuestro cuerpo en sí mismo, y del cuerpo vivido, que se refiere a la forma en que nuestra corporalidad se manifiesta en nuestras relaciones humanas y en la socialización.

Nuestros cuerpos no son sólo el lugar desde el cual llegamos a experimentar el mundo, sino que a través de ellos llegamos a ser vistos en él (MerleauPonty, 1976: 5). Para, Merleau-Ponty él yo esta ubicado en el cuerpo, que a su vez está ubicado en el tiempo y en el espacio. A veces somos conscientes de nuestros cuerpos como objetos que se han de mirar, en espacios sociales concretos, mientras que en otros, como el hogar, no sintonizamos con nuestros cuerpos como objetos que han de ser contemplados. En los espacios públicos podemos sentir que estamos en primer plano, mientras que, cuando estamos en casa, nos encontramos entre bastidores. La vestimenta es siempre "contextual", es decir, que se adapta a situaciones muy distintas. Puede suceder que haya algunos momentos en los que el acto de vestirse constituya un acto irreflexivo, similar a ir de compras o a recoger a los niños al colegio y otros, en que el acto de vestirse es consciente y reflexivo, como vestirse para una entrevista de trabajo o una reunión importante.

El tiempo y el espacio ordenan nuestro sentido del yo en el mundo, nuestras relaciones y encuentros con los demás y, también, la forma de cuidar de nuestros cuerpos. En Occidente, la práctica cotidiana de vestirse implica ser consciente del tiempo, porque la moda ordena la experiencia del yo y del cuerpo en el tiempo, y esta ordenación del tiempo se da especialmente a través del sistema de la moda que se organiza en dos temporadas: primavera-verano y otoño-invierno. Las revistas de moda, a su vez, congelan el flujo de las prácticas cotidianas del vestir y lo ordenan en distintas categorías: pasado, presente y futuro. La sociología del vestir y las prácticas de la industria de la moda pueden utilizar estos términos fenomenológicos para ver cómo la experiencia de cuidar y presentar el cuerpo está social y temporalmente constituida.

El espacio es la otra dimensión de nuestra experiencia del cuerpo y de la identidad $^{5}$. El espacio es externo para los individuos, en cuanto impone reglas y normas particulares sobre ellos, e interno para los mismos, en cuanto es expe-

5. Mientras Merleau-Ponty considera que nosotros captamos el espacio externo a través de la situación corporal o esquema corporal o postural; por su parte Foucault contempla al espacio en relación con el orden social. 
rimentado y, de hecho, transformado por ellos ${ }^{6}$. Los espacios públicos (la calle, la oficina y los centros comerciales) funcionan con normas distintas y determinan cómo hemos de presentarnos y cómo hemos de interactuar con los demás. El espacio también es experimentalmente distinto según la hora del día. Otro aspecto importante a tener en cuenta es que la acción transforma el espacio: franquear el espacio es sortear objetos y personas. En este sentido, el espacio es tanto social como sensorial. Aunque Merleau-Ponty sea bueno al describir el espacio y su percepción, Goffman proporciona explicaciones concretas sobre cómo ocurre esto en el mundo social. Esta definición del espacio como movimiento estructuralista y la presentación del yo como algo que los individuos tienen que captar e interpretar es valiosa para una explicación del vestir como práctica corporal contextuada. El vestir forma parte del orden microsocial de la mayoría de los espacios sociales, y cuando nos vestimos hemos de tener presente las normas implícitas de dichos espacios, ya que existe un código del vestir que hemos de cumplir. Puede que no siempre seamos conscientes, quizá sólo en ciertas circunstancias, como en las situaciones formales, que exigen un alto grado de conciencia corporal y del vestir. Sin embargo, interiorizamos ciertas reglas o normas del vestir que utilizamos inconscientemente a diario.

Los espacios también tienen género. Las mujeres experimentan los espacios públicos de modo muy distinto a como los experimentan los hombres. Según Entwistle (1997), el espacio impone sus propias estructuras en las personas, que, a su vez, pueden idear estrategias de vestir encaminadas a controlar ese espacio. Así, es más probable que la mujer profesional sea más consciente de su cuerpo y del vestir en los espacios públicos laborales que en el hogar. Los espacios laborales tienen distintos significados para las mujeres y ellas han desarrollado estrategias especiales de vestir para controlar las miradas de los demás, sobre todo las de los hombres. Del estudio de Tseelson (1997) también se desprende que el grado más alto de autoconciencia del aspecto se manifestaba en ocasiones muy formales como en las bodas o en entrevistas de trabajo. Su investigación demuestra de qué modo el aspecto y, por ende, la indumentaria está sujeta a diversos grados de conciencia según la situación. Las mujeres más que los hombres ven sus cuerpos como objetos a los «cuales se ha de mirar»; al efecto podemos afirmar que la conciencia del aspecto corporal está influida por el género. Las mujeres suelen identificarse más con el cuerpo que los hombres, y eso puede generar experiencias de corporeidad diferenciales: se puede afirmar que las mujeres tienen más tendencia a desarrollar una mayor conciencia corporal de ellas mismas como un ser corpóreo que los hombres, cuya identidad no está tan situada en el cuerpo.

6. En el mundo social existe un orden moral que impone en los individuos formas correctas e incorrectas de presentarse y vestirse. En este aspecto, el trabajo de Goffman debe mucho a las ideas de Durkheim, que dijo que la vida social no sólo está funcionalmente ordenada, sino también moralmente regulada. Crossley (1995a) considera que Goffman lleva el análisis de la conducta corporal más lejos que Mauss o Merleau-Ponty. 


\section{El cuerpo «habla»}

La mayoría de los estudios sobre el comportamiento humano en los procesos de interacción, hasta hace nada, se dirigían sobre todo a la comunicación verbal. Sin embargo, a comienzos de la década de 1960 se abre un nuevo campo de investigación: la comunicación no verbal. De esta forma, el cuerpo se presenta como una estructura lingüística que "habla» y revela infinidad de informaciones aunque el sujeto guarde silencio. $\mathrm{Al}$ parecer, «hablamos con nuestros órganos fonadores, pero conversamos con todo nuestro cuerpo" (D. Abercrombie, 1968: 55) ${ }^{7}$. La escuela de Palo Alto ha profundizado particularmente en los aspectos no verbales de la comunicación, esto es, en la imposibilidad de permanecer sin comunicar. La nueva comunicación ya no es una simple relación entre dos personas en la que una envía la pelota a otra, como en una partida de ping-pong, sino que se concibe como un sistema cultural en el que se inserta el individuo, sistema regido por una causalidad que ya no es lineal, sino circular, donde el efecto retro actúa sobre la causa. Esta nueva forma de comunicación se comporta como una orquesta, donde cada miembro forma parte y en la que todo el mundo sigue una partitura invisible. Partitura polifónica, ya que la comunicación se produce a niveles distintos (verbal, corporal, gestual, espacial, aspecto externo...) y a veces contradictorios (Gregory Batenson, 1990). En este sentido, la comunicación se define como cualquier intercambio de información que se verifique en el interior de un sistema de relaciones, con independencia del medio que se utilice para comunicar y del hecho de que los interlocutores tengan o no conciencia de ello.

Birdwhistell (1979), partiendo de la lingüística estructural y de la teoría cibernética de la interacción (feedback), propone una nueva aproximación teórica de los «micromovimientos» corporales. A este estudio cultural comunicacional de los movimientos corporales le denomina Kinesia. Observo que, de igual modo que el lenguaje hablado puede descomponerse en sonidos, palabras, oraciones, en la Kinesia existen también unidades similares, la menor de ellas es el kine (movimiento apenas perceptible) y, por encima de éste, existen otros movimientos mayores y más significativos llamados Kinemas, portadores de un gran sentido y significado cuando se les toma en conjunto e incluso son intercambiables. Al parecer, existen entre cincuenta o sesenta kinemas para todo el cuerpo, y más del 33\% están concentrados en el rostro. Sin embargo, es la cultura la que otorga un significado a cada uno de los innumerables movimientos corporales posibles. Como todos los movimientos del cuerpo no se podían analizar a simple vista, este autor creó un sistema taquigráfico, cuyo signo es el kine, que permite el «microanálisis» de los movimientos corporales, su resultado es parecido a la partitura de un

7. D. ABERCROMBIE. «Paralanguage», en British Journal of Disorders of Comunication, 3, Londres, 1968 , p. 55. Sobre este tema, véase la selección de estudios a cargo de R. HiNDE, La comunicazione non verbale, Bari, Laterza, 1974, así como la obra de M. ARGYLE, Il corpo e il suo linguaggio, Bolonia, Zanichelli, 1982. 
director de orquesta; una vez terminada la trascripción, se procede a verificar las regularidades corporales.

Otro de los elementos analizados es la proxémica, ésta es la ciencia que estudia el uso del espacio y la distancia corporal como una señal que regula las interacciones sociales. Sin entrar en más detalles sobre esta teoría, Edward Hall (1987), en su libro La dimensión oculta, distingue varias zonas dentro del uso del espacio entre los cuerpos: la distancia intima (reservada para aquéllas personas a quienes se les permite el contacto corporal), social (es la que suele mantenerse en las situaciones normales de interacción) y publica (preservada para aquellos que actúan ante una audiencia). En la interacción, cuando la distancia íntima y personal es invadida, la gente siempre trata de recuperar su espacio y al efecto envía una mirada fija al intruso (Goffman) que le indicará que se aparte.

Erving Goffman $(1971,1979,1991)$ parte de la idea que para que la interacción social sea viable, se necesita información acerca de aquéllos con quienes se interactúa. Y entre las muchas vías por las que los actores pueden adquirir dicha información es tanto a través de lo que dicen y hacen como de la apariencia. En su teoría, un elemento importante es la «fachada personal» (front), que define como «la dotación expresiva empleada por el individuo durante una actuación, ya sea de forma intencional o no». Así, al comienzo de cualquier encuentro, es importante distinguir algunas de las características más sobresalientes del otro, con el fin de saber cómo comportarse. Los distintos componentes del aspecto externo adquieren importancia sobre todo por su significado social, es decir, por los mensajes más o menos manipulados, que están en relación con él yo y que consiguen comunicar algo. Tales informaciones no son necesariamente verdaderas, pero es suficiente que expresen lo que el individuo desea que los demás piensen de él para que sean confirmadas. La presentación de nosotros mismos mediante señales no verbales, mediante lo que Erving Goffman llama "glosario del cuerpo" (body gloss) es, sin duda, tanto una de las señales no verbales que más influye en las percepciones, tanto en las reacciones de todos los individuos en general como en las de cada uno en particular. Así, es necesario invertir en el cuerpo volviendo al gimnasio, ya que el cuerpo, junto a su envoltorio, es el primer signo mediador en la nueva relación social; pues es aquello con lo que nos presentamos. El cuerpo habla por sí solo y la palabra enmudece.

Actualmente, en las grandes ciudades, ha surgido una nueva subcultura relacionada con los procedimientos comunicativos a los que recurren sus usuarios, es especialmente a través de los diversos lenguajes no verbales. La elaboración de lenguajes en los que predominan los aspectos meta comunicativos (gestos, posturas, ademanes, vestimenta, aditamentos, etc.) sobre los comunicativos, conduce a posibilitar interacciones en donde predomina la comunicación acerca de la relación que mantienen entre sí los comunicantes, sobre las interacciones en donde predominan las comunicaciones acerca de los contenidos. Con estas premisas, el cuerpo, del que hemos hablado, se configura como un procedimiento calificador de otras informaciones, entre las que des- 
tacan la propia identidad personal, el indicador del grupo, la adscripción y la pertenencia a determinados grupos. En la nueva cultura, el cuerpo es una señal que vincula, separa u oculta la particular forma y las condiciones en las que cada individuo se adscribe y pertenece a una clase social, a una edad, a un sexo, a una profesión, a una actividad, a un contexto determinado y a un espacio.

\section{El cuerpo como mercancía y signo}

En la sociedad de consumo, el cuerpo se transforma en mercancía y pasa a ser el medio principal de producción y distribución. Su mantenimiento, reproducción y representación se convierten en temas centrales. El análisis que Jean Baudrillard (1974) realiza sobre el cuerpo en la sociedad de consumo es interesante por su impacto sociológico. En primer lugar, considera que la lógica social del consumo es una lógica de consumo de signos, donde el cuerpo aparece dentro del abanico de los objetos de consumo, y bajo el signo de la liberación sexual, el cuerpo comienza a ser objeto de numerosas inversiones narcisistas, físicas y eróticas. Todo ello prueba que el cuerpo se ha convertido en un objeto de salvación. Y se constituye un proceso de «sacralización» sobre el cuerpo como valor exponencial. Pero lo más importante en todo este proceso es que el cuerpo parece haber sustituido al alma como objeto de salvación. La propaganda y la publicidad se encargan continuamente de recordarnos que tenemos un solo cuerpo y que hay que salvarlo y cuidarlo. Para Baudrillard, el cuerpo funciona según las leyes de la «economía política del signo», donde el individuo debe tomarse a sí mismo como objeto, como «el más bello de los objetos" psíquicamente poseído, manipulado y consumido para que pueda instituirse en un proceso económico de rentabilidad. Lo que Baudrillard ha querido demostrar en su análisis es que las estructuras actuales de producción y consumo proporcionan al individuo una doble representación de su cuerpo: como una forma de capital y como fetiche, es decir, el cuerpo moderno se exhibe como una forma de inversión y signo social a la vez (Baudrillard, 1974: 185-213). Si antaño el alma envolvía el cuerpo, hoy es la piel la que lo envuelve, pero como un signo de prestigio y de referencia. Mientras en la sociedad contemporánea el estatus general de la propiedad privada se aplica igualmente al cuerpo, a la práctica social y a la representación mental que de él se tiene. Por el contrario, en la sociedad tradicional, no hay percepción espectacular de su cuerpo, sino una visión instrumental mágica, inducida por el proceso de trabajo y la relación con la naturaleza.

Las transformaciones acaecidas desde los años setenta hasta la actualidad han obligado a los teóricos sociales a replantearse el peso de los especialistas en ciencias humanas y sociales, quienes, en íntima relación con el poder, el sistema de producción y de consumo de masas, han contribuido a conformar los actuales usos sociales del cuerpo. En las sociedades occidentales, las dietas alimenticias, los excesos y los defectos dietéticos se han ido consolidando. Featherstone (1990, 1991, 1992, 1995), en sus artículos sobre el cuerpo y el ejercicio físico, investiga el modo en que se experimenta el cuerpo en la cultura contemporánea 
de consumo. Y arguye que desde principios del siglo XX ha habido un espectacular aumento en los regímenes de autocuidado del cuerpo ${ }^{8}$. El cuerpo se ha convertido en el centro de un trabajo cada vez mayor a través del ejercicio, la dieta, el maquillaje y la cirugía estética, y hay una tendencia general a ver el cuerpo como una parte del propio yo que está abierto a revisión, cambio y transformación. El crecimiento de los estilos de vida sanos son testimonios de esta idea de que nuestros cuerpos están inacabados y son susceptibles de cambio. Al parecer, no nos contentamos con ver el cuerpo como una obra completa, sino que intervenimos activamente para cambiar su forma, alertar sobre su peso y su silueta. El cuerpo se ha convertido en parte de un proyecto en el que hemos de trabajar, proyecto que va vinculado a la identidad del yo de una persona. El cuidado del cuerpo no hace referencia sólo a la salud, sino también a sentirse bien; nuestra felicidad y realización personal, cada vez más, están sujetas al grado en que nuestros cuerpos se ajustan a las normas contemporáneas de salud y belleza. Featherstone distingue dos tipos de exigencias con respecto al cuerpo: que el cuerpo interior (inner body) funcione bien (esté sano y en forma) y que la apariencia (outer body) sea cuidada. Así, el cuerpo es un «signo», es un «mensaje» que habla de su propietario. Ya no es importante ser feliz y encontrarse bien consigo mismo, sino la proyección que tienen los demás sobre uno mismo.

Por otra parte, en las últimas décadas se plantea la cuestión de conocer cómo las diferencias biológicas entre los individuos se transforman en factores de discriminación social. En este sentido, diversos problemas de salud actuales chocan con la definición de «cuerpo ideal». La gordura es un buen ejemplo para comprender cómo las modas sociales utilizan una categorización de enfermedad, etiquetando lo "normal» en un momento dado como "patológico», en otro. La exclusión social de las personas obesas, el rechazo a la obesidad tiene tal relevancia que debería ser objeto de un análisis sociológico más exhaustivo que desbordaría esta investigación, de momento sólo queremos resaltar que se estigmatiza a personas cuyo peso no es un riesgo para su salud ni un obstáculo para su vida social porque choca con la definición de "cuerpo ideal». Esta presión por el ideal estético se ha ido intensificando. No obstante, no todos los obesos están igualmente estigmatizados. El cuerpo ideal es mucho más importante para la mujer que para el hombre. Por ello, las mujeres obesas están más discriminadas socialmente y presionadas para hacer dieta que los varones obesos y son ellas las que llenan las clínicas de control de peso (Alemany, 1993: 45-52).

Mientras en las sociedades desarrolladas se consolidan cada vez más las dietas, en cuyo ascetismo encontramos un impulso agresivo con respecto al cuerpo; por el contrario, en las sociedades antiguas practicaban rituales de ayuno, cuya función es la de expurgar y reabsorber, a través de la observación colectiva, todo el impulso agresivo que se desarrolla hacia el cuerpo. Nuestra sociedad

8. J. Toro y sus colaboradores en España (1989) hicieron un análisis de la publicidad «pro esbeltez» incluida entre las 10 revistas «femeninas» con más de 100.000 ejemplares editados que se publican en España y observaron que casi uno de cada cuatro anuncios invitaba directa o indirectamente alcanzar un peso más bajo. 
de consumo excluye por principio toda norma restrictiva por ser incompatible con la liberación del cuerpo, de forma que todo impulso agresivo recae hoy en la más profunda preocupación por lo físico. Así, más allá de la moda, el cuerpo se convierte en un objeto que hay que despertar, llevar a sus justas medidas y mortificar con fines estéticos, con los ojos fijos en las modelos esqueléticas y demacradas de cualquier revista de moda.

Otro conjunto de análisis sobre los usos sociales del cuerpo en las sociedades contemporáneas provienen de la sociología francesa con los trabajos de L. Boltanski (1971: 205-223). Este autor define el «hábito corporal» de los miembros de un grupo como un sistema de normas muy arraigadas, que aunque no se expresen en su totalidad de forma sistemática, organizan implícitamente la relación de los individuos del mismo grupo social con sus cuerpos, de forma que su cultura somática presenta una unidad profunda. El cuerpo, al igual que otros objetos, marca la posición de los individuos en la jerarquía social, es un signo de estatus mayor cuanto que no es percibido como tal.

A su vez, P. Bourdieu (1986, 1998, 1991) ofrece un análisis sociológico de la corporeidad potencialmente útil. Sus trabajos intentan desarrollar una dialéctica entre las estructuras sociales, por una parte, y el agente, por otra. En La teoría de la distinción (1998) integra el cuerpo y sus diferentes usos en un esquema explicativo global, donde relaciona, de un modo determinista, las condiciones sociales de existencia, el habitus y las prácticas; "el gusto" y el "estilo de vida». Lo primero que hace es elaborar un espacio social objetivo y lo estructura en tres compones básicos de la clase social: el "capital económico», el «capital cultural» y el «capital social», a través de los cuales determina las distintas prácticas de los estilos de vida diferenciados. Al aplicar su análisis a la manera de tratar el cuerpo, de moldearlo y de nutrirlo, observó que los espacios de preferencias corporales (alimenticios, higiénicos y deportivos) se organizan según el propio volumen del capital económico, cultural y social, donde la propia actitud corporal es reveladora del habitus y, en consecuencia, de la clase social de pertenencia. El gusto corporal dependerá de la idea que cada clase se hace del cuerpo, de las categorías que emplea para evaluarlo y de los efectos que utiliza sobre el mismo. El gusto corporal contribuye hacer el cuerpo de clase. El cuerpo es una de las formas más objetivas de expresar el gusto de clase, tanto a través de su apariencia, de sus dimensiones, como en la manera de tratarlo. Y todo ello es revelador de las disposiciones más profundas del habitus ${ }^{9}$. $\mathrm{El}$ habitus es, por consiguiente, un concepto que vincula al individuo con las estructuras sociales. El modo en que vivimos en nuestros cuerpos está estructurado por nuestra posición social en el mundo y por nuestra clase social. Todas las agrupaciones de clase tienen su propio habitus, sus propias disposiciones que son adquiridas mediante la educación, tanto formal como informal, a través de la familia, la escolarización y similares.

9. El concepto de habitus es «un sistema de disposiciones duraderas y transportadoras que son producidas por las condiciones particulares de una agrupación de clase social» (1994: 95). 
El gusto es una manifestación obvia del habitus y, tal como parece indicar la propia palabra gusto, es una experiencia tremendamente corpórea. El gusto forma parte de las disposiciones corporales de una agrupación de la clase social, los gustos por comidas especiales, por ejemplo, el caviar, se dice que son «adquiridos», es decir, que son aprendidos, desarrollados o fomentados y son indicativos de la posición social; de este modo, el potencial del habitus como concepto para pensar desde la óptica de la corporeidad es que el modo en que llegamos a vivir en nuestros cuerpos está estructurado por nuestra posición social en el mundo, pero estas estructuras son reproducidas únicamente mediante las acciones materializadas de los individuos.

P. Bourdieu (1986) considera que el cuerpo es «un producto social», en el sentido de que de él se hace siempre una lectura social: entre cuerpos «distinguidos» y cuerpos «vulgares». Él dibuja el espacio social de los cuerpos de clase, dejando de lado los azares biológicos, ya que en el cuerpo se tiende a reproducir la estructura del espacio social y su trayectoria. La soltura o torpeza de movimientos corporales no se reparte por igual entre las distintas clases sociales, ambas resultan de la comparación entre el cuerpo real y el cuerpo ideal. Cuanto menor sea la diferencia percibida entre los dos cuerpos, tanto más probable es que se experimente la ligereza y seguridad características de los agraciados por la figura perfecta. Esta afortunada sensación suele prodigarse más entre los miembros de las clases dominantes. Por el contrario, la probabilidad de sentir el cuerpo bajo el desagradable signo de la incomodidad, la torpeza o la timidez crece con la desaprobación percibida entre el cuerpo real y el cuerpo soñado. No es extraño que nuestra constitución corporal se vea afectada por nuestra posición social. La talla, el peso, el desarrollo muscular, la soltura o torpeza de movimientos y gestos, los hábitos alimentarios, la higiene, entre otras formas de expresión corporal, se ven afectadas o influidas por nuestra posición social.

\section{Cuerpos, ciencia y tecnología}

En la actualidad, el cuerpo ya no puede considerarse como algo fijo (o una identidad fisiológica), sino que ha acabado profundamente implicado en la reflexibilidad de la modernidad ${ }^{10}$. En las sociedades tradicionales, el cuerpo solía considerarse un aspecto de la naturaleza, regido sólo incidentalmente por la intervención humana. No obstante, todo esto se vio alterado por la progresiva invasión del cuerpo por sistemas abstractos o los conocimientos especializados ${ }^{11}$. El cuerpo, al igual que el yo, pasa a ser un lugar de interacción, apropiación y

10. En relación con la reflexibilidad social, cfr. GIDDENS (1992), El capitalismo y la moderna teoría social, Labor, Barcelona.

11. Giddens (1995), en general, define a los sistemas abstractos como las representaciones simbólicas. A su vez, por sistemas expertos entiende sistemas de conocimiento especializado de cualquier tipo que depende de reglas de procedimiento trasferibles de un individuo a otro (p. 297). 
reapropiación. Si al principio se creyó que era el lugar del alma y, más tarde, el centro de necesidades oscuras y perversas, el cuerpo es ahora plenamente susceptible de ser trabajado por las influencias de la modernidad reciente. A consecuencia de estos procesos, se han alterado sus límites (Giddens, 1995: 275).

Una de las características de la modernidad es «el reconocimiento de que la ciencia y la tecnología tienen un doble filo y crean unos parámetros de riesgo y peligro, al tiempo que ofrecen posibilidades beneficiosas para la humanidad» (Giddens, 1995: 43). El progreso científico y médico genera unas soluciones técnicas que cambian el significado del cuerpo. Estas técnicas han permitido que tras la muerte de una persona se puedan donar sus órganos y seguir viviendo en otro cuerpo, o pueden dejar una parte de su material genético en espera de que algún día una mujer logre una gestación.

El nacer y el morir son dos experiencias que hasta hace poco tiempo marcaban los límites del tránsito terrenal de todo ser humano. El nacimiento suponía el inicio de una identidad social de un ser humano y la muerte era el fin. En el momento en que el corazón se paraba, se consideraba que todos los órganos vitales habían dejado de funcionar y la persona pasaba a ser un cadáver, un cuerpo inerte, sin vida. La definición del principio y del fin del cuerpo humano determinada por las leyes biológicas ha ido dejando de ser una condición inamovible con el consecutivo avance de la medicina. Gracias a las nuevas tecnologías médicas, el alcance de los expertos raya lo que hace pocos años se consideraba ciencia-ficción. Al poder modificar las condiciones que definen el inicio o el fin del cuerpo, se crean unas posibilidades en el ámbito de algunas enfermedades mortales y de la reproducción, pero lo más significativo es que se genera la necesidad de redefinir la relación entre el cuerpo humano y la identidad individual.

Los dos tipos de procedimientos tecnológicos (los trasplantes de órganos y la reproducción asistida), aunque muy distintos, comparten algunas características fundamentales: primera, que los trasplantes de órganos y las NTRA ${ }^{12}$ tienen en común que se extraen de una parte del cuerpo del individuo al cual pertenecen (un órgano, esperma u óvulos) que pueden continuar ejerciendo sus funciones y desarrollándose en otro cuerpo. Y, segunda, que los procedimientos requieren de la participación de amplios grupos sociales, sin los cuales sería difícil conseguir los órganos para realizar trasplantes o el material genético necesario para la inseminación artificial o la fecundación in vitro ${ }^{13}$.

Los profesionales médicos juegan un papel destacado legitimando y difundiendo las nuevas tecnologías, tanto en el espacio político como en la sociedad. La finalidad es doble: por una parte, la aceptación de la práctica y, por otra, y como consecuencia, la obtención de órganos, esperma y óvulos. En todo este proceso los profesionales se convierten en redistribuidores de cuerpo

12. Las tecnologías de la reproducción asistida acercan cada vez más la ciencia al deseo de creación de la vida.

13. Respecto a una redefinición del nacimiento y la muerte, ver «El cuerpo humano ante las nuevas tecnologías médicas» (1994), REIS, no 68, p. 173-195. 
y contribuyen así a una redefinición del inicio y el fin de la vida humana. Se trata de un discurso muy controvertido. Tanto las tecnologías de la reproducción asistida como los trasplantes de órganos necesitan, para su aplicación, nuevas categorías del cuerpo, de la identidad y de la existencia. Por una parte, las tecnologías de la reproducción asistida redefinen el inicio de la vida, del proceso de creación y de las relaciones filiativas. Por otra, las técnicas del trasplante de órganos dan lugar a una nueva definición de la muerte, de la identidad del cuerpo y de sus partes, así como de los límites de la vida. Las nuevas tecnologías son ejemplos de la capacidad de la ciencia y de la medicina, para construir imágenes sociales y culturales. Son exponentes del progresivo poder sobre el cuerpo y la vida.

Lo que está ocurriendo forma parte de lo que A. Giddens $(1995,1998)$ denomina la "socialización de la naturaleza», expresión que hace referencia al hecho de que ciertos fenómenos que antes eran «naturales», o que venían dados por la naturaleza, ahora tienen un carácter social, es decir, que dependen de nuestras propias decisiones. La reproducción humana es un ejemplo de ello. Durante siglos, la vida de las mujeres estuvo determinada por el parto y la crianza de los hijos. En la época premoderna, la anticoncepción no era efectiva y en algunas sociedades no se conocía. Incluso en la Europa del siglo XVIII, era habitual que las mujeres tuvieran hasta veinte embarazos (con abortos frecuentes y muertes de niños $\left.{ }^{14}\right)$. La mejora de los métodos anticonceptivos ha trasformado esta situación de forma significativa. En los países industrializados no es «natural» tener tantos embarazos, es algo que apenas ocurre. Los avances en los métodos anticonceptivos propician que las mujeres y los hombres puedan decidir si quieren (o no) descendencia. La prevención del embarazo es sólo un ejemplo de las técnicas de reproducción ${ }^{15}$.

A medida que se desvincula la reproducción de la especie, ésta pasa a ser una decisión individual cuyos efectos resultan incalculables. ¿Qué elegirán los padres cuando puedan diseñar a sus hijos y qué límites habrá que poner a esa capacidad de elección? Probablemente, la ingeniería genética no será barata. ¿Qué significará esto, que todo aquél que pueda pagarlo programará a sus hijos de forma que carezca de las características físicas que se consideren socialmente indeseables? ¿Qué ocurrirá con los hijos de los grupos más desfavorecidos, que seguirán naciendo de forma natural ${ }^{16}$. No hay un ejemplo mejor de la mezcla de oportunidades y problemas que la creciente socialización de la naturaleza.

El posible impacto social de la ingeniería genética ${ }^{17}$ está comenzando a suscitar un debate casi tan intenso como el que rodea al problema del aborto.

14. Ver la Historia del control de los nacimientos de Alfred SAUvy (1972).

15. Anthony Giddens (1998), Sociología, Alianza, Madrid, p. 167.

16. En relación con los debates sobre las tecnologías de la reproducción asistida, véanse Testard (1996), Spallone y Steinberg (1987), Testard (1993), Charlesworth (1993) y Dick (1995).

17. En relación con las cuestiones suscitadas por la ingeniería genética humana, véanse Keyles y Hood (1992), Andrews y otros (1994) y Borrillo (1996). 
Los que están a favor de este tipo de manipulación afirman que nos aportará muchos beneficios. Por ejemplo, será posible identificar los factores genéticos que hacen que algunas personas sean más vulnerables a ciertas enfermedades. La reprogramación genética garantizará que algunas dolencias no se transmitan de generación en generación. Será posible «diseñar» nuestro cuerpo antes del nacimiento.

La ciencia como fuerza transformadora de cambio social sitúa a la sociedad ante un conjunto de enigmas y dilemas valorativos. El dilema ético más polémico que han planteado las nuevas técnicas de reproducción en las sociedades contemporáneas es el aborto: ¿en qué condiciones debería serles posible abortar a las mujeres? La polémica se ha hecho tan intensa precisamente porque se centra en problemas éticos fundamentales que no tienen una solución fácil. Los que están "a favor de la vida» creen que el aborto está mal siempre, excepto en circunstancias extremas, porque equivale a un asesinato. Para ellos, las cuestiones éticas están sujetas, por encima de todo, al valor que hay que conceder a la vida humana. Los que están «a favor de la elección» señalan que el control de la madre sobre su propio cuerpo, su derecho a disfrutar de una vida gratificante, debe ser la principal consideración. La polémica ha generado numerosos episodios violentos. ¿Es posible que se resuelva alguna vez? Al menos un destacado experto en teoría jurídica y social, Ronald Dworkin (1993), ha señalado que sí es posible. Según este autor, las profundas divisiones que existen entre los que subrayan el derecho a la vida y los que recalcan el de la elección ocultan las razones aún más profundas que hay para el acuerdo entre los dos bandos y que, por tanto, puede haber ciertas esperanzas. En otras épocas de la historia, la vida valía relativamente poco. Sin embargo, en la actualidad, nos hemos acostumbrado a otorgarle un carácter sagrado. Los dos bandos están de acuerdo con ello, pero lo interpretan de forma diferente; uno hace hincapié en los intereses del niño y otro en los de la madre. Según Dworkin, si se logra convencer a ambos bandos de que comparten un mismo sentido ético, será posible establecer un diálogo más constructivo.

En relación con el poder médico, hemos de plantearnos la siguiente cuestión: cuando se ha pasado de un cuerpo prohibido (en la sociedad tradicional) a un cuerpo instrumental (en la sociedad industrial) a un cuerpo racional (en la sociedad actual o en las sociedades avanzadas); cuando las sociedades contemporáneas se caracterizan por el poder y el control de la natalidad, la fecundidad in vitro, la posibilidad de elegir el sexo; es muy probable que el control médico en el siglo XXI se vaya a caracterizar por el control y el poder de los individuos de elegir el momento de desprenderse de su cuerpo: de decidir la hora de su muerte. Pues cuando nuestros cuerpos son cuerpos para las relaciones, el individuo no quiere estar o ser prisionero de su propio cuerpo, sino que quiere librarse de su propio cuerpo, como es el caso de los enfermos terminales y los tetrapléjicos. Ahí, desde nuestro punto de vista, estará la nueva frontera de los cuerpos y el gran debate del siglo XXI.

Se entiende por eutanasia (del griego eu y thanatos; literalmente, 'buena muerte') el conjunto de acciones u omisiones que, de un modo deliberado y 
consciente, provoca, o permite, un curso de acontecimientos conducente a poner fin a la vida de una persona por el bien de ésta, bien porque ella así lo ha decidido, bien porque en los casos en los que no puede elegir por sí misma entre la vida y la muerte, otros han tomado la decisión por ella. La eutanasia toma la forma de asistencia al suicidio o de suspensión de los tratamientos médicos para el mantenimiento de la vida, en la inteligencia de que es preferible la muerte a continuar una existencia de sufrimiento insoportable o a mantener una vida carente de aprecio por sus condiciones de deterioro ${ }^{18}$. Las decisiones sobre la vida y la muerte son las más cruciales, tanto por su extraordinaria relevancia moral como por su complejidad. De ahí que asuntos como el de la eutanasia o el aborto resulten muy controvertidos y cada cual trate de fundamentar sus opiniones en aquellas razones, filosóficas, morales o religiosas, que considera más profundas e irrenunciables. De hecho, la regulación jurídica de la eutanasia produce debates que dan lugar a una verdadera colisión entre el ámbito moral y el jurídico. Algunos, incluso, han llegado a ver en estas controversias ciertas analogías con las guerras de religión de hace trescientos años $\left(\right.$ Dworkin $\left.{ }^{19}\right)$. Es cierto que la mayoría de las diferentes culturas, grupos y personas consideran a la vida humana algo sagrado, pero el valor de la vida humana y su centralidad moral se interpretan de muy distinta manera. De ahí que la eutanasia plantee verdaderos dilemas morales entre el valor primordial de la vida humana y el respeto por la autonomía de la persona, entre la defensa de un principio tan arraigado como el de la «santidad» de toda vida humana y la disposición benevolente a procurar una vida valiosa y evitar el sufrimiento.

El cuerpo humano está programado genéticamente para morir. Pero, ¿hasta cuándo? Si los expertos en genética encuentran un modo de controlar el envejecimiento y la muerte, éste sería uno de los aspectos más importantes de la socialización de la naturaleza antes mencionada. Los científicos ya han demostrado que células madres pueden manipularse para hacerlas actuar como células jóvenes. Ronald Katz, presidente de la Academia Estadounidense de Medicina contra el Envejecimiento, ha señalado: "Creo que vamos a ver vidas mucho más largas, quizá en el curso de la nuestra. Ya disponemos de nuevas tecnologías, sólo tenemos que desarrollarlas. Hay que empezar a prepararse para una sociedad sin edades. El envejecimiento es una enfermedad que puede tratarse» (Kelsey, 1996: 2) ${ }^{20}$.

Por conflictivo que pueda resultar, y a pesar de las fragilidades de la argumentación jurídica actual ${ }^{21}$, considera que el cuerpo es para el derecho una

18. Cfr. Giner, S.; Lamo, E. y otros, Diccionario de Sociología (1998), Alianza Editorial, p. 281-282.

19. DwORKIN, R.M. (1994), El dominio de la vida: una discusión acerca del aborto, la eutanasia y la libertad individual, Barcelona, Ariel.

20. Kelsey, Tim (1996), "I want to live for ever», Sunday Times News Review, 7 de enero de 1996, p 2.

21. BorRILLO, D., «Estatuto y representación del cuerpo humano en el sistema jurídico», REIS, no 68 , p. 211-222. 
cosa y que el lazo jurídico que une a la persona (sujeto de derecho) con su dimensión corpórea (objeto de derecho) es una relación de tipo «derecho subjetivo». Esta constatación no hace más que afirmar lo que venía sucediendo históricamente. Los avances científicos han puesto de manifiesto aquello que de manera más o menos oculta existía en el orden jurídico. Podríamos lamentarnos de que, en lugar de inspirarse en las teorías de filosofía existencialistas o de psicoanálisis para pensar la persona, el derecho haya optado por la opción más clásica del dualismo, reconociendo que no es el cuerpo lo que le interesa para definir la personalidad, sino la razón y la voluntad. Asimismo, parece contradictorio que sea un derecho análogo al de la propiedad quien califique la relación que el hombre mantiene con su cuerpo. Lo que resultaría inadmisible es negar estas realidades que han forjado la reflexión jurídica (o la falta de ella) acerca del cuerpo.

El sistema jurídico fundado en la distinción entre las personas y las cosas reconoce que el cuerpo es una cosa, pues las partes separadas de él son cosas. A partir de esta constatación, podemos encontrar matices, pues, como vemos, para el derecho estadounidense el cuerpo es una mercancía, en tanto que para el sistema francés es una cosa fuera del comercio. Vemos que, respondiendo a dos tradiciones diferentes pero condicionados por una filosofía común, los sistemas jurídicos estadounidense y francés acuerdan otorgar al cuerpo humano la calidad de cosa. Las tradiciones que lo diferencian son aquéllas que han marcado las respectivas familias jurídicas. Así, el derecho anglosajón hará de la noción de propiedad privada el eje central en torno al cual gira el régimen de protección de la persona. Es justamente porque goza de un derecho de propiedad inalienable en que el individuo puede disponer de su cuerpo con la libertad con la que dispone de otros bienes. El sistema francés, heredero del régimen romano, encuentra dificultades para pensar la persona física en tanto que corpórea. Pero los avances de la ciencia lo ponen frente a una realidad incontestable: las partes y los elementos del cuerpo se pueden conservar independientemente del individuo del que provienen. Ellos son considerados cosas porque el cuerpo es una cosa. Tal calificación no implica considerar al cuerpo como una mercancía. Se trata de una cosa fuera del comercio y cuya disponibilidad está condicionada a fines superiores que no dependen sólo de la libertad individual. Por ello, en el derecho francés, a diferencia del derecho estadounidense, la relación que une la persona a su cuerpo no es tanto un derecho de propiedad, sino más bien un derecho subjetivo relativo ${ }^{22}$.

A pesar de las diferencias enunciadas, subyace una filosofía común a ambos sistemas: el dualismo cartesiano. En efecto, de la misma manera que en el pensamiento racionalista el cuerpo sólo puede concebirse a partir del cogito, para el derecho la persona abstracta es titular de un elemento instrumental (el cuerpo), del que podrá disponer en mayor o menor medida según optemos por

22. La noción de «derecho subjetivo» es central en el pensamiento jurídico moderno, designa la facultad del sujeto o el poder individual de la voluntad. 
uno u otro sistema legal. Como dijimos antes, la idea de propiedad es aquí una noción compleja que incluye todos los problemas de la definición de una persona. En el ámbito de la política de la vida, este problema recoge las cuestiones de cómo habrá de elegir el individuo en relación con las estrategias del desarrollo corporal en el planteamiento de su vida y quien determinará la disposición de los productos y las partes del cuerpo. En este asunto, como tan a menudo ocurre en el terreno de la política de la vida, aparecen unidos problemas de definición filosófica, derechos humanos y moralidad que la sociedad tendrá que resolver.

\section{A modo de conclusión}

En la actualidad, el cuerpo está sujeto a fuerzas sociales de una índole bastante distinta al modo en que se experimenta en las comunidades tradicionales. Por una parte, los discursos contemporáneos sobre la salud y la imagen vinculan al cuerpo y a la identidad y sirven para promover ciertas prácticas de cuidados corporales típicas de la sociedad contemporánea. Por otra parte, el cuerpo se ve fragmentado por los avances de la ciencia y de la tecnología y la creciente «socialización de la naturaleza y de la reproducción». La reproducción siempre había estado en uno de los polos de la implicación del hombre con la naturaleza y en el otro se hallaba la muerte. No obstante, a través de los avances de la ingeniería genética, el hombre puede cortar el lazo que conecta la vida de la especie con el de la reproducción como proceso natural. La ciencia sitúa a la sociedad ante un conjunto de dilemas (aborto, eutanasia, clonación) difíciles de resolver.

\section{Bibliografía}

ABERCROMbie, D. (1968). «Paralanguage». British Journal of Disorders of Comunication, 3. Londres.

Aisenson Kogan, A. (1981). Cuerpo y persona. México: FCE.

AlEMANY, M. (1993). Obesidad y nutrición. Madrid: Alianza.

ANDREWS, L.; FullarTON y otros (eds.) (1994). Assessing Genetic Risk. Implications for Health and Social Policy. Washington (DC): National Academy Press.

ARIÉs, Philippe (1987). El niño y la vida familiar en el Antiguo Régimen. Madrid: Taurus. BAÑUELOS, Carmen (1994). «Los patrones estéticos en los albores del siglo XX». REIS, no 68, p. 119-140, octubre-diciembre. Madrid.

BAUdrillard, Jean (1974). La sociedad de consumo. Barcelona: Plaza \& Janés.

BATENSON, Gregory; BIRDWHISTELL, R.L. y otros (1990). La nueva comunicación. Barcelona: Kairos.

BECK, Ulrich (1998). La sociedad del riesgo. Hacia una nueva modernidad. Barcelona: Paidos.

BELL, Daniel (1991). El advenimiento de la sociedad post-industrial. Madrid: Alianza. - (1994). Las contradicciones culturales del capitalismo. Madrid: Alianza Universidad. Benthall, J. (1976). The Body Electric: Patterns of Western Industrial Culture. Londres:

Thames and Hudsson. 
Berger, P. (1980). Modos de ver. Barcelona: Gustavo Gili.

BERGER, P.; LuCKMANN, T. (1968). La construcción social de la realidad. Buenos Aires: Amorrortu.

Berthelot, J.M. «Sociological Discourse and the Body». En Featherstone, M.; HePworTh, M.; Turner, B. (comps). The Body: Social Process and Cultural Theory. Londres: Sage.

BIRDWHISTELL, R.L. (1972). Kinesics and context. Essays on body motion comunication. University of Pennsylvania Press: Filadelfia.

- (1979). El lenguaje de la expresión corporal. Barcelona: Gustavo Gili.

BOLTANSKI, Luc (1971). «Lesusages sociaux du corps». Annales d'Economie. Société et civilisation, $\mathrm{n}^{\mathrm{o}} 1$, enero/febrero, vol. 26, p. 205-223. Está traducido en (1975): Los usos sociales del cuerpo. Buenos Aires: Periferia.

Borrillo, D. (1994). "Estatuto y representación del cuerpo humano en el sistema jurídico». REIS, no 68, p. 211-222.

- (ed.) (1996). Genes en el estrado. Madrid: Consejo Superior de Investigaciones Científicas.

BOURDIEU, Pierre (1986). «Notas provisionales sobre la percepción social del cuerpo». En VArela, Julia (eds.). Materiales de sociología crítica. Madrid: La Piqueta.

- (1988). "Espacio social y Poder simbólico». En Cosas dichas. Madrid: Gedisa.

- (1991). El sentido de las prácticas. Madrid: Taurus.

- (1998). La distinción, criterio y bases sociales del gusto. Madrid: Taurus.

- (2000). La dominación masculina. Barcelona: Anagrama.

BRAIN, R (1979). The Decorated Body. Londres: Hutchinson.

BroHM, Jean Mari (1975). Corps et politique. París: Delarge, Coll. Corps et Culture. - (1982). Sociología política del deporte. México: FCE.

BuÑUEL, Ana (1994). "La construcción social del cuerpo de la mujer en el deporte». REIS, no 68, p. 97-117.

Charlesworth, M. (1993). Bioethics in a Liberal Society. Cambridge: Cambridge University Press.

ClARK, Kenneth (1981). El desnudo corporal. Madrid: Alianza.

Colectivo del Libro de Salud de las Mujeres de Boston (1984). Nuestros cuerpos nuestras vidas. Barcelona: Icaria, p. 85-94.

Crossley, N. (1995). «Body Techniques, Agency and Inter-Corporality On Goffman's Relations in Public». Sociology, vol. 129, no 1, p. 133-149.

- (1995b). "Merleau-Ponty, the Elusive Body and Carnal Sociology». Body and Society, vol. 1, p. 43-63.

- (1996). «Body/Subjet, Body/Power: Agency, Inscription and Control in Foucault and Merleau-Ponty». Body and Society, vol. 2, p. 99-116.

CSORDAS, T.J. (1993). «Somatic Modes of Attention». Cultural Antropology, vol. 8, $\mathrm{n}^{\circ} 2$, p. $135-156$.

- (1996). «Introduction: The Body as Representation and Being-in-the-World». En CSORDAS, T.J. (comp.). Embodiment and Experience: The Existential Ground of Culture and Self. Cambridge: Cambridge University Press.

DAVIS, Flora (1973). La comunicación no verbal. Madrid: Alianza.

Dossey, L. (1992). Tiempo, espacio y medicina. Barcelona: Kairos.

Douglas, Mary (1979a). "Do Dogs Laugh”. A Cross Cultural Approach to Body Symbolism». En Douglas, M. (comp.). Implicit Meanings: Essays in Anthropology. Londres: Routledge.

- (1979b). Implicit Meanings: Essays in Anthropology. Londres: Routledge. 
- (1988). Simbolos naturales: exploraciones en cosmología. Madrid: Alianza.

- (1991). Pureza y peligro: análisis de los conceptos de contaminación y tabú. Madrid: Siglo XXI.

DurÁn, Ma Ángeles (1988). De puertas adentro. Madrid: Ministerio de Cultura, Instituto de la Mujer, Serie Estudios, no 12.

Dyck, J. Van (1995). Manufacturing Babies and Public Consent Debating the New Reproductive Techologies. Londres: Macmillan.

DwORKIN, Ronald (1994). El dominio de la vida. Una discusión acerca del aborto, la eutanasia y la libertad individual. Barcelona: Ariel.

ELIAS, Norbet (1988). El proceso de la civilización. Madrid: FCE.

ENTWISTLE, Joanne (2002). El cuerpo y la moda. Barcelona: Paidos.

- (1997). Fashioning the Self: Woman, Dress, Power and Situated Bodily Practice in the Workplace. Tesis doctoral, University of London, Goldsmiths' College.

FalK, P. (1994). The Consuming Body. Londres: Sage Publications.

Featherstone, M. (1990). «Perspectives on consumer culture». Sociology, no 24, p. 5-22.

- (1991a). "The Body in a Consumer Society». En Featherstone, M.; Hepworth, M.; Turner, B. (comps). The Body: Social Process and Cultural Theory. Londres: Sage.

- (1991b). Consumer Culture and Postmodernism. Londres: Sage Publications.

Featherstone, M.; Turner, B. (1999) «Introduction». Body and Society, vol. 1, no 1, 1995.

FEHER, M.; NADDAFF, R.; TAZI, N. (eds.) (1989). Fragments for a History of the Human Body. 3 vols. Nueva York: Zone. [(1991). Fragmentos para una historia del cuerpo humano. 3 vols. Madrid: Taurus.]

FLANDRIN, Jean-Louis (1984). La moral sexual en occidente. Barcelona: Granica.

FouCAult, Michel (1980). La microfísica del poder. Madrid: La Piqueta.

- (1995). Historia de la sexualidad. 3 vols. 1, Madrid: Siglo XXI.

- (1996). Las tecnologías del yo y otros textos afines. Barcelona: Paidos.

- (1999). El nacimiento de la clínica: una arqueología de la mirada médica. Madrid: Siglo XXI.

- (2000). Vigilar y castigar. Madrid: Siglo XXI.

Frank, A.W. (1990). «Bringing Bodies Back In». Theory, Culture and Society, vol. 7, no 1. GAlimberTi, Umberto (1993). II Corpo. Milina, Universale Economica, Feltrinelli. GIDDENS, Anthony (1995). Modernidad e identidad del yo: el yo y la sociedad en la época contemporánea. Barcelona: Península.

- (1998). Sociología. Madrid: Alianza.

GIL CALVO, Enrique (2000). Medias miradas. Un análisis cultural de la imagen femenina. Barcelona: Anagrama.

GofFman, Erving (1971). Ritual de la interacción. Buenos Aires: Tiempo Contemporáneo.

- (1979). Relaciones en público. Micro estudio del orden público. Madrid: Alianza.

- (1987). La presentación de la persona en la vida cotidiana. Buenos Aires: Amorrortu.

- (1991). «La ritualización de la feminidad». En Los momentos y sus hombres. Barcelona: Península.

Hall, Edward T. (1987). La dimensión oculta. Enfoque antropológico del uso del espacio. Madrid: IEA.

INGLEHART, Roland (1991). El cambio cultural en las sociedades industriales avanzadas. Madrid: Centro de Investigaciones Sociológicas. 
Irigaray, Luce (1977). Ce sexe quin 'en est pas un. París: Minuit.

- (1992). Yo, tú, nosotras. Madrid: Cátedra.

Johnson, M. (1991). El cuerpo en la mente. Madrid: Debate.

Kevles, D.J.; Hood, L. (eds.). (1992). The Code of Codes. Scientific and Social Issues in the Human Genome Project. Cambridge (MA): Harvard University Press.

LAquer, T.; Bourgois, (1992). Corporal Politics. Cambridge, MA: MIT List Visual Arts Center (citado en ENTWISTLE, J. (2002). El cuerpo y la moda. Una visión sociológica. Barcelona: Paidos, p. 27).

Laquer, T.; Gallagher, C. (1987). The Making of the Modern Body: Sexuality Society and The $19^{\text {th }}$ Century. Londres: University of California Press.

LASCH, Ch. (1981). La cultura del narcisismo. Madrid: Alianza.

LEE BARTKY, Sandra (1994). «Foucault, feminismo y modernidad del poder patriarcal».

En Mujeres, derecho penal y criminología. Madrid: Siglo XXI, p. 197-210.

LEONI, D.; BERTET, T. (1995). Las cifras record del cuerpo humano. Barcelona: Paidotribo. LIPOVETSKY, Gilles (1986). La era del vacío. Barcelona: Anagrama.

MAuss, Marcel (1973). "Techniques of the Body». Economy and Society, vol. 2, no 1, p. 70-89.

- (1991). «Técnicas y movimientos corporales». En Sociología y antropología. Madrid: Tecnos, p. 338 y s.

MARKIDES, K.S.; COOPER, C.L. (eds.) (1987). Retirement in Industrialized Societies. Social Psychological and Heath Factors. Chichester: John Wiley.

McNay, L. (1992). Foucault and Feminism: Power, Gener and the Self. Cambridge: Polity Press.

- (1999). "Gender, Habitus, and the Field-Pierre Bourdieu and Limits of Reflexivit». Theory, Culture and Society, vol. 16, no 1, p. 95-117.

Merleau-Ponty, M. (1976). The Primacy of Perception. Evanston y Chicago: Northwestern University Press.

- (1981). The Phenomenology of Perception. Londres: Routledge and Kegan Paul (trad. al castellano: Fenomenología de la percepción. Barcelona, Planeta (1985).

Polhemus, T. (1988). Bodystyles. Lutton Lennard.

- (1994). Streetstyle. Londres: Thames and Hudson.

Polhemus, T.; Proctor, L. (1978). Fashion anti-Fashion: An Antropology of Clothing and Adornment. Londres: Cox and Wyman.

RAMAZANOGLU, C. (comp.) (1993). Up against Foucault: Explorations of some Tensions betweens Foucault and Feminism. Londres: Routledge.

SAUVY, Alfred (1972). Historia del control de los nacimientos. Barcelona: Península. ScotT, S.; Morgan, D. (1993). Body Matters. Essays on the Sociology of the Body. Londres: The Falmer Press.

SENNET, Richard (1997). Carne y piedra. El cuerpo y la ciudad en la civilización. Madrid: Alianza.

ShILling, Chris (1993). The Body and Social Theory. Londres: Sage.

Spallone, P.; SteInberg, D.L. (eds.) (1987). Made to Order. The Myth of Reproductive and Genetic Progress. Oxford: Pergamon Press.

SQUICCIARINO, Nicola (1990). «Las principales señales no verbales». En El vestido habla: consideraciones psico-sociológicas sobre la indumentaria. Madrid: Cátedra, p. 17-24. SynnotT, A. (1993). The Body Social: Symbolism, Self and Society. Londres: Routledge. TeSTARD, J. (1993). La procreación artificial. Debate, 1994.

TORO, Josep (1996). El cuerpo como delito: anorexia, bulimia, cultura y sociedad. Barcelona: Ariel. 
Tseelson, E. (1997). The Masque of Feminity: Londres: Sage.

TURNER, Bryan (1989). El cuerpo y la sociedad: exploraciones en la teoría social. México: FCE.

- (1994). «Avances recientes en la teoría del cuerpo». Revista Española de Investigaciones Sociológicas, $\mathrm{n}^{\circ}$ 68, p. 11-40.

VARELA, Julia (1989). Sujetos frágiles. Ensayos de sociología de la desviación. Madrid: FCE, p. 17-52.

WeBer, Max (1969). La ética protestante y el espíritu del capitalismo. Barcelona: Península. Welton, D. (ed.) (1998). Flesh and Body. Massachussets: Blackwell Publishers.

WiLSON, Eduard (1980). Sociobiología, La nueva sintesis. Barcelona: Omega.

Wolf, Naomi (1991). El mito de la belleza. Barcelona: Emecé. 